\title{
Induction of the McCollough effect I: Figural variables
}

\author{
KAREN G. FOREIT and BRUCE A. AMBLER \\ University of Texas at Arlington, Arlington, Texas 76019
}

\begin{abstract}
The effect of line orientation and line configuration on the induction of orientation-specific negatively colored aftereffects was investigated in three separate studies. In the first study, subjects viewed magenta-and-black vertical gratings with one eye, alternating with green-andblack vertical gratings to the other. Monocular tests revealed complementary aftereffects in each " eye which disappeared when the test patterns were viewed with both eyes together. In Study 2, imposing a single colored bar against a black background induced negatively colored aftereffects in a white bar against a black background and in a black-and-white grating, while imposing a single black bar against a colored background was ineffective. In Study 3, presenting a magenta square outline elicited green aftereffects in vertical and horizontal bars and gratings as well as in outlines of squares and diamonds, while pairing the magenta square with a green cross had no effect. It was concluded that the induction mechanism responsible for the McCollough effect is sensitive to line orientation but not to shape. This specificity appears incompatible with a simple conditioning model.
\end{abstract}

McCollough (1965) reported that exposing subjects to orange-and-black vertical gratings and blue-andblack horizontal gratings led to reports of the complementary colors in achromatic test gratings. These colored aftereffects (CAEs) were specific to the induction orientation, appearing even in the vertical and horizontal portions of radiating lines and spirals, and lasted for an hour or more. Succeeding researchers (see Skowbo, Timney, Gentry, \& Morant, 1975) continued to use gratings as inducing and test stimuli, which led to the parametric investigation of grating characteristics such as spatial frequency. While this line of research has proved fruitful, focusing on repetitive patterns such as gratings has somewhat obscured the contribution of figural variables such as orientation, contrast, and figuregroup relationships which can be found in simple, nonrepeated figures as well. The purpose of the present research was to investigate the effects of these figural variables.

The confounding of figural (e.g., orientation) and repetitive (e.g., spatial frequency) variables becomes especially important when one examines the theoretical explanations that have been offered to explain the McCollough effect. McCollough (1965) suggested that the orientation specificity of the aftereffect arose from selective adaptation of colored edge detectors, similar to those described by Hubel

This research was supported by a grant from the Organized Research Fund of the University of Texas at Arlington to the authors. We would like to thank Rebecca Beckham and Margaret Bruton for their assistance in collecting data. We would also like to thank Drs. Lorrin Riggs, John Uhlarik and Keith White for their comments on earlier versions of this paper. Reprint requests can be mailed to Bruce Ambler at Department of Psychology, University of Texas at Arlington, Arlington, Texas 76019. and Wiesel (1962). The majority of Hubel and Wiesel's detectors receive binocular input, so it might be expected that monocular adaptation would affect the unadapted eye as well. Furthermore, adaptation of strictly figural variables appears to show such interocular transfer (see Skowbo et al. 1975). However, numerous reports in the literature (see Skowbo et al. 1975), as well as unpublished work in our own laboratory have failed to produce interocular transfer of the McCollough effect. Presenting a sequence of colored grids to one eye elicits no colored aftereffects in the unexposed eye.

The failure to obtain interocular transfer does not completely rule out the role of binocular mechanisms in the consolidation of the McCollough efect. When White and Riggs (1974) presented half a colored chevron figure to each eye, some subjects observed an aftereffect when the complete figure was viewed monocularly. They concluded that the aftereffects for angles could be mediated by a cortical mechanism receiving input from both eyes. If such a mechanism is critical for induction, we should fail to observe an aftereffect or observe a weakened aftereffect if mutually incompatible information is introduced to the two eyes. McCollough's (1965) original paper included the finding that antagonistic effects (orange vertical, blue horizontal vs. orange horizontal, blue vertical) could be sequentially induced, one in each eye. Since her report included no data, we decided to replicate this finding, using simultaneous rather than successive induction of the two eyes.

\section{STUDY 1}

\section{Method}

Subjects. Subjects were recruited from introductory psychology courses requiring experimental participation. They were screened 
for family history of color blindness and had either normal visual acuity or correction with contact lenses. Eight women and three men participated in Study 1.

Materials. Induction slides consisting of magenta and greeen filters (Wratten 53 and 26 , respectively) paired with achromatic square-wave gratings were projected by a Kodak Carousel slide projector onto a white cardboard in front of the subjects. Overall luminances of the induction figures were $4.09 \mathrm{~cd} / \mathrm{m}^{2}$ (magenta) and $5.07 \mathrm{~cd} / \mathrm{m}^{2}$ (green). The projected gratings measured $90 \mathrm{~cm}$ square. The subjects sat between 1.2 and $2.7 \mathrm{~m}$ from the wall, and, depending on the subject's seating location, a single cycle of the grating subtended a visual angle of $1.88^{\circ}$ to $1.17^{\circ}$.

Test stimuli, as shown in Figure 1, consisted of $5.08 \times 5.08 \mathrm{~cm}$ printed achromatic gratings in horizontal, vertical, and diagonal orientations as well as an unpatterned gray field. The horizontal and vertical gratings consisted of 11 black-white cycles, the diagonals of 15 cycles. The test pages were collected into a looseleaf booklet. The last page of this booklet extended beyond the test figures, and arranged on the extension were a series of Munsell chips ranging from highly saturated pink to highly saturated green; a number next to each chip indicated the saturation rating. The pink chips were given a positive sign, the green chips a negative sign. No color or gray was indicated by zero. The following Munsell colors and their ratings were used as reference standards: $5 R P 7 / 8(+8), 5 R P 7 / 4(+4), N / 7(0)$, 5GY 7/4 (-4), 5GY 7/8 $(-8) .^{1}$ The booklet also included a set of colored chips individually mounted on separate pages; these chips included the reference standards plus intermediate values: 5RP $7 / 6(+6)$, 5RP $7 / 2(+2)$, 5GY $7 / 2(-2)$, 5GY $7 / 6(-6)$. The saturation rating of each chip was printed on the back of the page. Test booklets were assembled as follows: 18 pages of color chips ( 2 of each chip, in random order), followed by the printed achromatic grids. The reference standards were clearly visible for every page.

Procedure. The subjects were tested in groups of two to five. They were seated at right-handed desks and asked to keep their heads and bodies upright and their booklets straight (a line was provided on each desk surface for that purpose). Ambient light during the pre- and postinduction phases was provided by diffused overhead cool white fluorescent fixures; luminance of the white bars on the printed test grating was $1.87 \mathrm{~cd} / \mathrm{m}^{2}$. These lights were extinguished during induction, when the only light source was the slide projector mounted behind and above the subjects.

The experiment was conducted in three phases-preinduction, induction, and postinduction. In the preinduction phase the use of the reference standards was explained and subjects rated the set of colored chips using the reference standards, first with the right eye and then with the left. The unused eye was covered with a plain, tie-on Bausch and Lomb eye patch. Feedback was provided by the correct answers appearing on the back of each page. The subjects then rated achromatic gratings with each eye individually, writing their answers in the spaces provided above and below the gratings (see Figure 1).

Induction consisted of alternating presentations of magentavertical and green-vertical gratings. Each grating was presented for $15 \mathrm{sec}$ at a time, with a 2 -sec black interval between stimuli; the subjects saw a total of 40 exposures, 20 of each color. So that each eye saw a different color, the subjects were asked to cover the unused eye with the ipsilateral hand, taking care not to touch the eye and to screen out all light. The experimenter instructed "change hands" during the interstimulus interval.

Following induction, the subjects gave two monocular ratings of each achromatic figure; again, the unused eye was covered with a patch. Order of test (right or left eye first) was counterbalanced across subjects. After completing the monocular ratings, the subjects rated the gratings with both eyes at once.
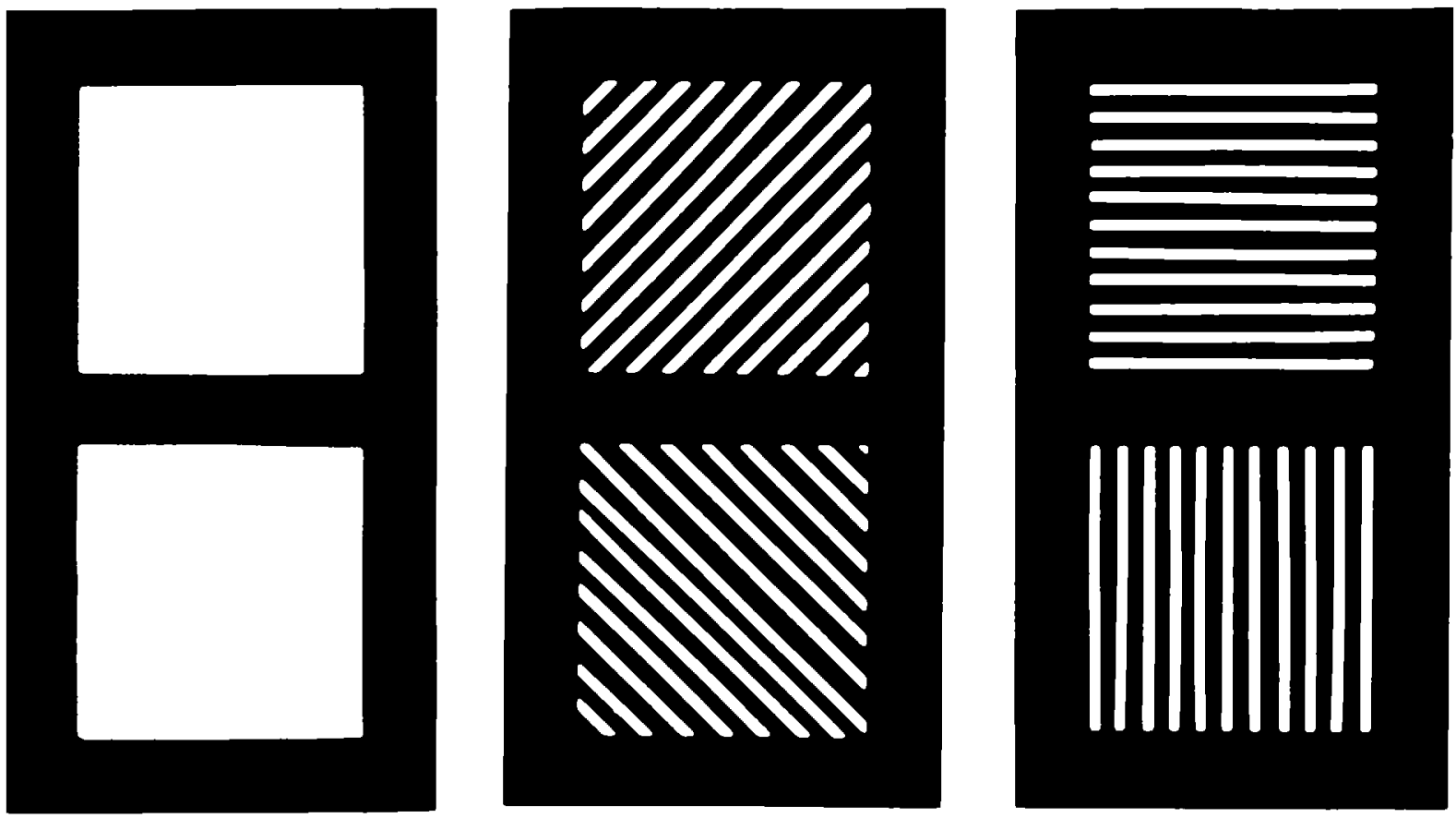

Figure 1. Achromatic test figures used to elicit afteref fects in Study 1. 


\section{Results and Discussion}

Data from subjects giving a rating of greater than \pm 1 to any achromatic figure in the preinduction phase were not included in the analysis. Three subjects failed to meet this criterion. The subjects' responses were recoded so that all negative aftereffects received a positive sign; thus a rating of -4 (phenomenal green) following exposure to magenta would be recorded as +4 , as would be a rating of +4 (pink) following exposure to green.

Each subject's mean rating for each test orientation was entered into a 2 (eye) by 3 (orientation: horizontal, vertical, diagonal) analysis of variance. This analysis indicated significant main effects of eye and test orientation $[F(1,7)=5.80, p<.05 ; F(2,14)$ $=22.15, \mathrm{p}<.01$, respectively]. The eye which had been exposed to magenta yielded a stronger effect, and more importantly, the aftereffects were largely confined to the induction orientation. Figure 2 presents the mean ratings given under the monocular tests to the three test orientations. Notice that the original sign of the ratings has been restored, indicating that the two eyes were seeing different (and complementary) colors. The binocular test was not included in this analysis because virtually no color was reported in this condition. Only two subjects reported seeing any color when viewing with both eyes; the group means for the vertical, horizontal, and diagonal orientations were 0.19 , 0.06 , and 0.0 , respectively.

These results are consistent with an inference of functional independence of the two eyes in inducing the McCollough effect. By utilizing a dichoptic viewing procedure, it was possible to induce mutually antagonistic aftereffects in the two eyes, which yielded a net result of no color under binocular viewing conditions.

Although we obtained a McCollough effect in a situation in which a binocular mechanism was not responsible for the effect, it is still possible that such a mechanism might be operational in other

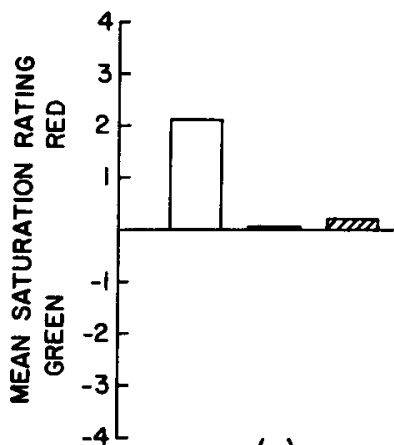

(a)

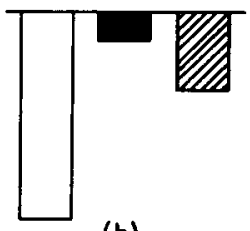

(b)
Figure 2. Mean aftereffects reported for monocular tests following dichoptic exposure to green and black vertical (a) and magenta and black vertical (b) gratings for the three test grating orientations. Reports of red are positive $(+)$ in sign; reports of green are negative $(-)$. situations. Thus, we might expect that the magnitude of the effect obtained in the present situation with incompatible colors would be less than would have been obtained had the colors been paired with orthogonal dimensions (e.g., magenta verticals to one eye, green horizontals to the other) or if subjects had seen only one color in one dimension (e.g., magenta verticals). Such a manipulation was not performed in Study 1, but data from other studies run in our laboratory under identical stimulus conditions fail to confirm this prediction. In Stúdy 1 , the mean rating of the aftereffect following exposure to magenta was -4.0 ; other subjects dichoptically viewing magenta and green paired with orthogonal gratings gave a mean rating of -3.2 following exposure to magenta (Ambler \& Foreit, 1978), and still other subjects viewing only magenta gratings also reported a mean rating of - 3.2 (Ambler \& Foreit, unpublished data). These three means $(-4.0,-3.2,-3.2)$ are not reliably different from one another, and indeed what difference does exist is opposite that predicted by the hypothesis that monocular input is combined centrally during induction. We conclude, therefore, that the contribution of eye-specific mechanisms far outweighs that of any binocular mechanisms that may exist.

One interpretation of this eye-specificity would be to link the ME with figural characteristics which can be analyzed by monocularly driven units and which do not require repeated patterns. Line orientation is one obvious example, given the usual reference to color-specific edge detectors. Furthermore, line orientation can be indicated just as easily with a single line as with a grating of many lines, either by a colored bar on black surround or a black bar on a colored surround. If CAEs can be linked with orientation, it should be possible to induce a McCollough effect with a single bar, and if orientation alone is sufficient to induce the ME, the colored bar in a black surround and the black bar in a colored surround should be equally effective. However, these two figures differ in contrast and figure-ground relationships, and if either of those factors is critical to the McCollough effect, we would expect a difference between the two stimuli. Contrast, for example, has been shown to be an important factor when repeated gratings are used (White, in press).

The color-bar/black-bar comparison also offers a way to test the classical conditioning model of the McCullough effect proposed by Murch (1976). In this model, the unconditioned stimulus (US) is the color of the induction slide and the response to be conditioned (CR) is a change in spectral sensitivity. The conditioned stimulus (CS) is the grating that appears with the color. Thus, presentation of magenta leads to greater relative sensitivity to green, and this change in sensitivity becomes linked to the orientation 
of the grating superimposed over the induction color. Murch supports this model by varying temporal parameters in a trace conditioning paradigm. Delaying onset of the color relative to onset of the grating reduces the magnitude of the aftereffect, as would be predicted by a conditioning model. Further support for the model is provided by Jones and Holding's (1975) report that testing accelerates the rate of decay. Presumably, eliciting the aftereffect by presenting achromatic gratings acts as extinction trials.

Another test of the conditioning model would be to vary the nature of the CS. The older models of classical conditioning (e.g., Hilgard \& Marquis, 1940) emphasize the importance of the US in establishing the CR and discount the nature of the CS as long as the organism is sensitive to it. Although more recent work (see Holland, 1977, for a review of the literature) has pointed to the importance of the CS in establishing the conditioned link, most of the studies showing superiority of one CS over another have compared different sensory modalities. Holland (1977) reported than when both CSs were drawn from the same modality there was either no difference between them in degree of conditioning or that any difference could be attributed to different levels of unconditioned responding. In the present context, the finding that one visual pattern successfully establishes the aftereffect while a comparable pattern does not would seriously question the conditioning explanation of the McCollough effect.

\section{STUDY 2}

\section{Method}

Subjects. Fourteen naive subjects, seven men and seven women, were recruited from the subject pool.

Materials. The magenta and green filters were combined with achromatic figures to produce two sets of induction stimuli: a single horizontal bar of magenta in a black surround and a single vertical bar of green in a black surround (colored bars); a single horizontal black bar in a green surround, and a single vertical black bar in a magenta surround (black bars). These slides were projected on a wall such that the bars subtended a visual angle of between $.94^{\circ}$ and $.58^{\circ}$, depending on the subject's seat; the colored surround in the black bar stimulus subtended a visual angle of between $13.32^{\circ}$ and $8.43^{\circ}$. The same luminances were used as in Study 1.

Test figures included the printed achromatic gratings used in the first study, plus horizontal, vertical, and diagonal orientations of white bars in black surrounds and of black bars in white surrounds. The new set of test stimuli is represented in Figure 3. These figures were gathered into test booklets similar to those used in Study 1.
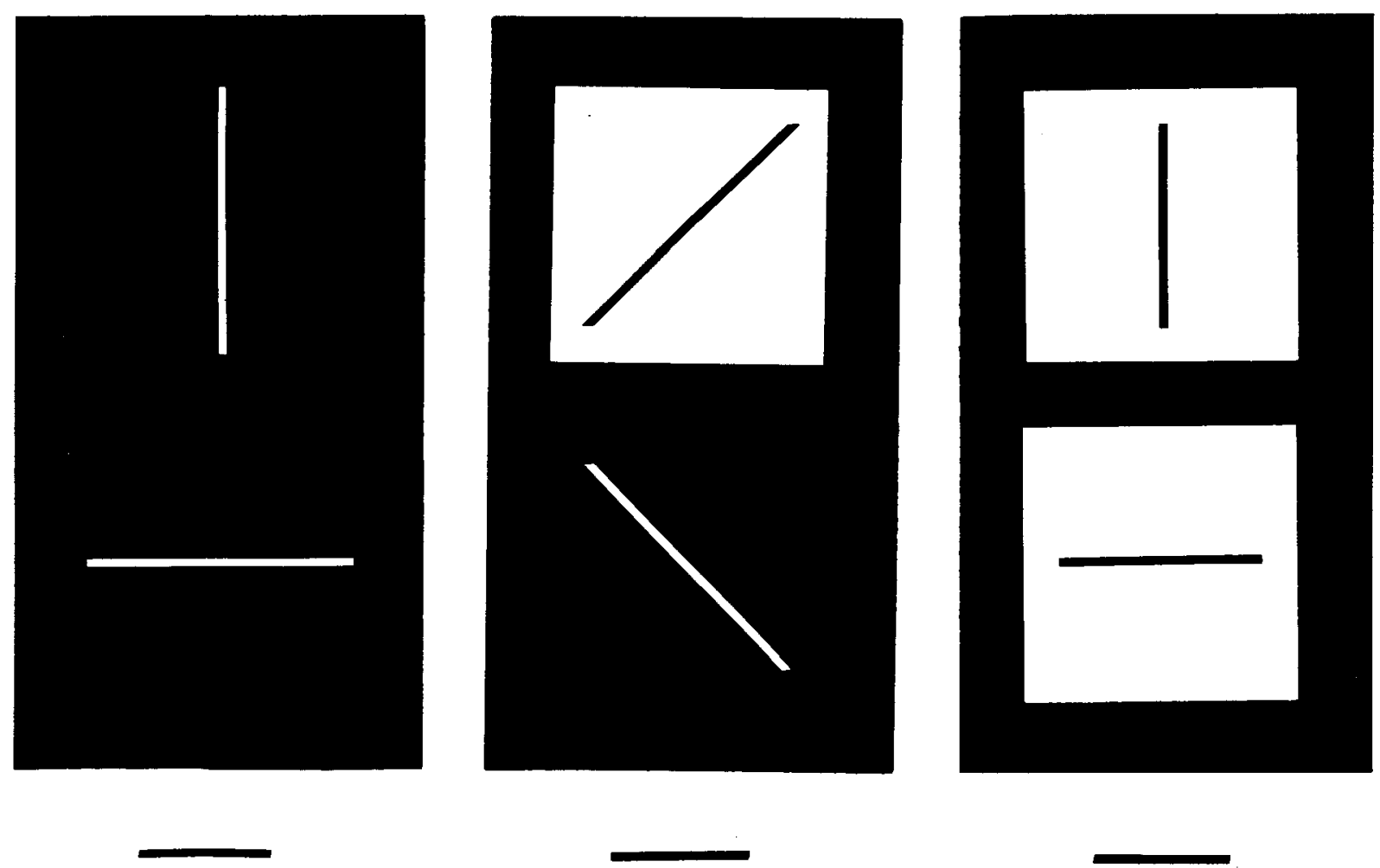

Figure 3. Achromatic test figures used to elicit aftereffects in Study 2. Gratings from Study 1 were also used (see Figure 1). 
Procedure. Pretraining and pretesting were accomplished as in Study 1. Each subject participated in both of two experimental conditions: colored bar, in which the colored bars in the black surround were presented, and black bar, in which the black bars in the colored surround were used. Induction and test were accomplished monocularly. The subjects were pretrained, pretested, induced, and tested in one experimental condition with one eye while the other eye was covered with an eye patch. Then the patch was moved to the previously uncovered eye, and the procedure was repeated. Order of treatments was counterbalanced across subjects.

\section{Results and Discussion}

The same pretest criterion for accepting data was used as in Study 1; two subjects failed to meet criterion. Neither experimental condition produced any measurable effect on the unadapted eye (counterbalancing the order of presentation permitted the second pretest to serve as a measure of interocular transfer).

The subjects' mean saturation ratings, recoded so that all negative aftereffects were positive in sign, were entered into a 2 (induction condition: colored bar vs. black bar) by 3 (test pattern: white bar on black, black bar on white, black-and-white grating) by 2 (test orientation: induced vs. diagonal) analysis of variance. All main effects and interactions proved statistically reliable. Induction with colored bars produced significantly higher ratings than induction with black bars $[F(1,11)=15.89, \mathrm{p}<.01]$, and the interactions with induction condition were a function of the uniformly low level of color reported under the black bar induction. Therefore, the effects of test pattern and test orientation will be discussed in context of the colored bar induction. The subjects gave their strongest color ratings when the tests matched the induction orientations $[F(2,22)=17.41$, $\mathrm{p}<.011$, seldom reporting any color at all in the diagonal orientations. Furthermore, these reports of color were almost totally confined to the white bar and grating test patterns $[\mathrm{F}(1,11)=14.34, \mathrm{p}<.01]$, leading to a reliable Test Pattern by Test Orientation interaction $[F(2,22)=9.65, p<.01]$. Follow-up $t$ tests of the colored bar induction group showed that the white bar and grating test patterns in the induction orientation elicited higher ratings than the black bar test pattern $[\mathrm{t}(11)=6.15, \mathrm{t}(11)=3.31$, for the white-bar/black-bar and grating/black-bar comparisons, respectively, $\mathrm{p}<.01$ in both cases], while the difference between the white bar and grating patterns was not statistically reliable [t(11) $=.50, \mathrm{p}>.10]$. The mean ratings elicited by the test patterns under both induction conditions are presented in Figure $4 .^{2}$ Since both pink and green aftereffects were elicited, all negative aftereffects, regardless of color, are positive in sign.

The results of Study 2 strongly question the role of classical conditioning in the induction of the McCollough effect. A single colored bar in a black surround successfully induced orientation-specific

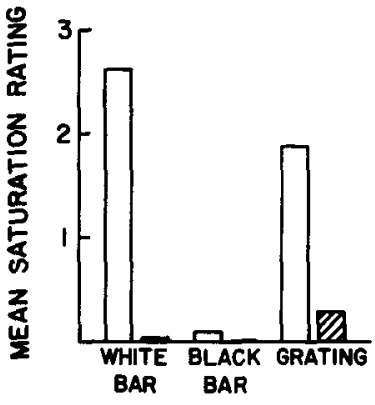

(a)
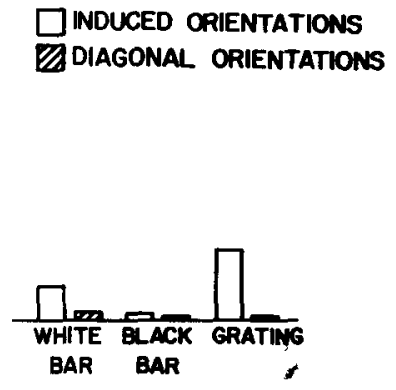

(b)
Figure 4. Mean aftereffects reported following induction with two different types of inducing stimuli: colored bars on a black background (a) and black bars on a colored background (b), for three types of test figures: white bar-black background, black bar-white background, achromatic gratings. All negativelycolored aftereffects, regardless of color, are scored as positive. The induced orientation is the mean of the horizontal and vertical ratings combined.

CAEs; a single black bar in colored surround did not. Similar stimuli were not equally effective as CS.

The success of the single bar is especially interesting. If CAEs may become associated with a single bar, they may also become associated with arrangements of bars. Put another way, can we demonstrate a McCullough effect not only for orientation but also for form.

Study 3 was designed to test this hypothesis by measuring the McCollough effect following exposure to geometric figures composed of straight horizontal and vertical lines. We reasoned that if the McCollough effect could be associated with mechanisms responsible for the perception of line arrangement as well as the lower-order orientation analyzers, complementary CAEs should be elicited by geometric figures which share the same line orientations and differ only in the arrangement of their constituent lines. A magenta square and a green cross were constructed from vertical and horizontal lines. These figures differed only in the arrangement of their component lines. If the McCollough effect can become associated with different line arrangements, CAEs should be induced by the alternation of these two figures. If the McCollough effect can only become associated with orientation differences, no CAEs should result from alternating the two figures. In either case, green aftereffects should be induced by presenting the magenta square above, since its component orientations and line arrangement both would be unopposed.

\section{STUDY 3}

\section{Method}

Subjects. Twenty subjects, thirteen women and seven men, participated. Another six potential subjects failed to meet the preinduction criterion. 
Materials. The magenta and green filters were combined with achromatic slides to produce an unfilled (line drawing) magenta square on a black surround and a green cross on a black surround. Bar width and total perimeter were identical for the two figures; thus, each of the four legs of the cross was identical in length to one side of the square. The projected widths of the square and cross were 45 and $90 \mathrm{~cm}$, respectively. Depending on the subject's seat, they subtended visual angles of $10.2^{\circ}-15.3^{\circ}$ (square) and $20.3^{\circ}-30.6^{\circ}$ (cross); the width of the colored bar subtended a visual angle of $.47^{\circ}-.75^{\circ}$

The achromatic test patterns included a blank white field against a black background, gratings in the vertical, horizontal, and diagonal orientations (Figure 1), a single white bar against a black background in the vertical, horizontal, and diagonal orientations (as in Figure 3), and a $4 \times 4 \mathrm{~cm}$ white outline of a square against a black ground presented upright as a square and rotated $45^{\circ}$ and presented as a diamond. Test booklets consisted of pages of colored chips to train subjects in the use of the rating scale followed by sections of the achromatic test patterns arranged in random order.

Procedure. The subjects were randomly divided into two experimental groups and tested in groups of two to five. For both experimental groups, pretesting, induction, and posttesting were accomplished monocularly; the unused eye was covered with a patch.

The subjects were trained on the use of the Munsell standards and rated the achromatic printed gratings with one eye at a time prior to any induction. During the induction phase, the subjects scanned the colored figures with the right eye. Subjects in the square-only induction condition viewed 4015 -sec exposures of the magenta square, with a 2 -sec black interstimulus interval. Subjects in the square-cross induction condition viewed $4015-\mathrm{sec}$ exposures of the magenta square alternating with 4015 -sec exposures of the green cross, again with a $2-\mathrm{sec}$ black interstimulus interval. Two minutes of dark adaptation folowed the induction phase. The subjects then rated each of the test fugures twice with each eye, one time through with the induced eye and once with the noninduced eye. Order of testing was counterbalanced across subjects.

\section{Results and Discussion}

Only the data from subjects meeting the preinduction criterion were included in the analysis. As in previous studies, there were virtually no reports of color from the noninduced eye; therefore the analysis of variance was restricted to reports of viewing with the induced eye.

The achromatic test patterns could be considered to belong in two categories: those presenting a single orientation (horizontal-vertical or diagonal) and those presenting a figure (square or diamond). For ease of analysis, subjects' ratings of the eight different patterns were consolidated into four test types: horizontal-vertical (mean of the ratings of horizontal and vertical gratings and single bars), diagonal (mean of the ratings of diagonal gratings and single bars), square (mean of the ratings of the square), diamond (mean of the ratings of the diamond). Since the measure of interest was the aftereffect of the magenta square, all reports of green were assigned a positive sign and all reports of pink were assigned a negative sign.

The data were then entered into a 2 (induction condition) by 2 (test pattern: single orientation vs. form) by 2 (test rotation: upright vs. diagonal) analysis of variance in which induction condition was a between-subjects variable and pattern and rotation were within-subjects variables. Because the unpatterned (blank) field elicited no consistent reports of color following either induction condition, it was not included in the analysis.

The analysis of variance revealed a highly significant main effect of induction condition $[F(1,9)=$ 13.22, $\mathrm{MSE}=3.82, \mathrm{p}<.01]$. As can be seen in Table 1, which presents the mean color ratings for each type of test pattern following the two induction conditions, the square-cross induction produced hardly any measurable colored aftereffects. The effect of rotation was not statistically reliable, but the Induction by Rotation interaction approached significance $[\mathrm{F}(1,9)=4.99, \mathrm{MSE}=.251, \mathrm{p}<.10]$. A planned comparison revealed a significant effect of rotation within the square-only condition $[F(1,9)$ $=13.98, \mathrm{p}<.01]$, which was entirely a function of higher reports of color in the horizontals and verticals than in the diagonals. As can be seen from Table 1, the square and the diamond elicited practically identical ratings. None of the other main effects or interactions approached statistical reliability. Finally, a planned comparison was performed on the color ratings elicited by the square test pattern following the two induction conditions. The critical prediction of the line-configuration hypothesis was that green aftereffects should be elicited by the magenta square under both the square-only and square-cross conditions. This comparison, based on the MSE of the triple interaction, revealed highly significant superiority of the square-only condition $[F(1,9)=40.20$, MSE $=.398$, $\mathrm{p}<.01$ ], caused by the total absence of green aftereffects in the square-cross condition.

These results fail to support the hypothesis that the ME can become associated with configurations of lines as well as the orientations of the individual components. Pairing a magenta square with a green cross failed to elicit reliable aftereffects. On the other hand, exposure to a magenta square alone induced an aftereffect that was more than an afterimage (no color was reported for an unpatterned field), that could be seen in individual lines and in

\section{Table 1}

Mean Chromatic Aftereffect Ratings Elicited by Exposure to a

Magenta Outline Square (Square Only) and by Exposure to a Magenta Outline Square Alternating with a Green Cross (Square-Cross)

Test Condition

\begin{tabular}{|c|c|c|c|c|}
\hline \multirow{4}{*}{$\begin{array}{l}\text { Induction } \\
\text { Condition }\end{array}$} & \multicolumn{4}{|c|}{ Test Condition } \\
\hline & \multicolumn{2}{|c|}{ Bars } & \multirow{2}{*}{\multicolumn{2}{|c|}{ Figures }} \\
\hline & \multirow{2}{*}{$\begin{array}{c}\text { Horizontal- } \\
\text { Vertical }\end{array}$} & \multirow[b]{2}{*}{ Diagonal } & & \\
\hline & & & Square & Diamond \\
\hline Square Only & -1.975 & -1.175 & -1.450 & -1.550 \\
\hline Square-Cross & +0.100 & +0.000 & +0.150 & -0.150 \\
\hline
\end{tabular}

Note-Green aftereffects are negative in sign, red aftereffects are positive. 
configurations of lines, and that was somewhat orientation-specific (the horizontal and vertical patterns elicited higher reports than the diagonals). However, the geometric figures and single lines elicited equivalent ratings, as did the square and diamond. The former result offers further evidence that the ME is associated with component orientations and not with configurations. The latter result was unexpected, and the present data offer no single interpretation.

\section{GENERAL DISCUSSION}

Studies 2 and 3 offer converging evidence for a number of issues. First, the validity of the classical conditioning model is seriously questioned. Not only can the McCollough effect not be elicited by very similar visual patterns (Study 2), but it can be eliminated entirely by pairing complementary colors with highly distinctive patterns with common constituent parts (Study 3). Furthermore, when the McCollough effect is successfully induced, it often fails to follow the kind of generalization gradient predicted by a conditioning mechanism. Exposure to a single colored bar in Study 2 showed greater generalization to gratings sharing the same orientation than to single bars in a different orientation, and the majority of aftereffects following black bar induction were made in response to white bar and grating test patterns, and not to the black bar which most closely resembled the inducing pattern. White and Riggs (1974) also reported cases in which a stronger CAE was elicited by new test patterns than by ones like the original stimulus.

Other conditioning models are still possible. For example, the critical link between pattern and color may lie in successive stimuli rather than within a single stimulus compound-perhaps it is the green following the magenta pattern that is important rather than the connection between the response properties of magenta and the pattern it appears with. Two findings render this model unlikely: first, only one color is necessary to induce a negative aftereffect. Second, work from our laboratory indicates that random presentations of the magenta and green pattern produce an effect just as strong as that induced by an orderly alternation of magenta and green. Clearly, interstimulus associations are not critical.

Second, the McCollough effect is sensitive to line orientation but not to form (line configurations). Orientation can be carried in single lines or patterns of lines, and color may be seen in single lines or patterns of lines that share orientational characteristics with the inducing stimulus. We have not been able to demonstrate an effect unique to a specific form which exists independently of a comparable effect in its constituent parts. Whatever higher-order form detectors may exist, they are either not colorspecific or incapable of becoming associated with color, thus accounting for the absence in the literature of CAEs dependent on "letters of the alphabet, people's faces, or makes of automobile" (White \& Riggs, 1974).

Third, figural properties are important in determining whether or not a McCollough effect is obtained. One important property could be fígureground characteristics. When colored gratings are used to induce a McCollough effect, the colored bars may be seen as colored figures against a black ground. In Study 2, the colored bar against a black background is seen as figure, while the black bar imposed on a colored background is also seen as figure but it is not colored. Reliable aftereffects can be produced by a colored-bar/black-background stimulus and by a color-and-black grating, but not by a black bar on a colored background; a feature common to both the colored grating and the colored bar may be the perception of color in the figure. Thus, color in the figure may be more important than color in the ground in inducing aftereffects.

Confounded with the figure-ground characteristics of the colored bar stimulus is brightness contrast. The colored bar on a black background provided more contrast than the black bar on a colored background, both directly in terms of ratio of black area to colored area and inversely in terms of distance between adjacent borders (width of colored area). If contrast is the critical stimulus determinant, we would expect that increasing the amount of contrast in the inducing pattern would increase the magnitude of the aftereffect. Results reported by Osgood (1974) can be interpreted in this way. Osgood manipulated the widths of the black bars and colored slits in his adaptation and test patterns and found that black bar width was the critical variable during the induction phase-the wider the black bar, the more pronounced the aftereffect. Wider black bars create greater contrast; the single colored bar in Study 2 represents the logical extreme of increasing black bar width.

Contrast and/or figure-ground relationships are necessary but not sufficient conditions for the induction of the McCollough effect, and they make themselves known only under appropriate colororientation conditions. As shown in Study 3, even when appropriate contrast and figure-ground relationships are maintained, no net aftereffect is observed when complementary colors are simultaneously paired with the same orientations.

In summary, we have reached three main con- 
clusions. First, figural properties of nonrepeated stimuli may exert strong influences on CAEs. Second, line configurations or forms per se are difficult to link with color to produce McCollough effects. And third, the classical conditioning model of Murch (1976) and other models based on conditioning fail to be supported by our findings.

\section{REFERENCES}

AMBler, B. A., \& Foreit, K. G. Induction of the McCollough effect II: Two different mechanisms. Perception \& Psychophysics, 1978, in press.

Hilgard, E. R., \& Marquis, D. G. Conditioning and learning. New York: Appleton-Century, 1940.

Holland, P. C. Conditioned stimulus as a determinant of the form of the Pavlovian conditioned response. Joumal of Experimental Psychology: Animal Behavior Processes, 1977, 3, 77-104.

Hubel, D. H., \& Wiesel, T. N. Receptive fields, binocular interaction, and functional architecture in the cat's visual cortex. Journal of Physiology, 1962, 160, 106-154.

Jones, P. D., \& Holding, D. H. Extremely long persistence of the McCollough effect. Joumal of Experimental Psychology: Human Perception and Performance, 1975, 1, 323-327.

McCollough, C. Color adaptation of edge detectors in the human visual system. Science, 1965, 149, 1115-1116.

MurCh, G. M. Classical conditioning of the McCollough effect: Temporal parameters. Vision Research, 1976, 16, 615-619.

OsGOoD, A. G. The role of bar width, slit width and spatial frequency of an orientation contingent color aftereffect known as the McCollough effect. Unpublished doctoral dissertation, Kansas State University, 1974.

Skowbo, D., Timney, B. N., Gentry, T. A., \& Morant, R. B. McCollough effects: Experimental findings and theoretical accounts. Psychological Bulletin, 1975, 82, 497-510.

Whute, K. D. Studies of form-contingent color aftereffects. In J. Armington, J. Kraskopf, \& B. Wooten (Eds.), Visual psychophysics: Its physiological basis. New York: Academic Press, in press.

White, K. D., \& Riggs, L. A. Angle-contingent color aftereffects. Vision Research, 1974, 14, 1147-1154.

\section{NOTES}

1. These particular Munsell values were selected by Osgood (1974) as the closest physical matches to subjects' aftereffects. We did not use the illuminant under which the Munsell scale was developed and so the subjects were not using a Munsell scale. However, the stimuli provided a hue and saturation range which was adequate to match the observed aftereffects.

2. The apparent CAEs induced by black bar induction are largely the results of the ratings made by one subject. Eliminating this subject reduces the mean saturation in the white bar test from .38 to .05 and the saturation in the grating test from .67 to .36 . Only 2 of the 12 subjects reported CAEs of greater than 1 (the maximum permitted preinduction rating) following black bar induction; in contrast, all 12 reported CAEs of greater than 1 following colored bar induction.

(Received for publication January 20, 1978; revision accepted May 4, 1978.) 